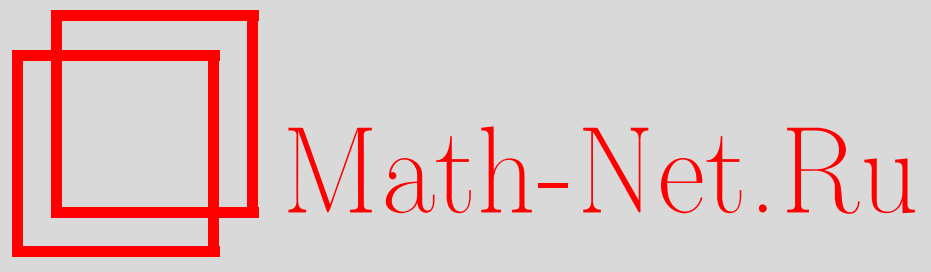

А. Н. Васильев, Исследование жидкокристаллических систем с примесями методами матричного формализма, TMФ, 2006, том 147, номер 2, 323-327

DOI: https://doi.org/10.4213/tmf1967

Использование Общероссийского математического портала Math-Net.Ru подразумевает, что вы прочитали и согласны с пользовательским соглашением http://www . mathnet.ru/rus/agreement

Параметры загрузки:

IP : 3.85 .7 .115

26 апреля 2023 г., 12:25:08

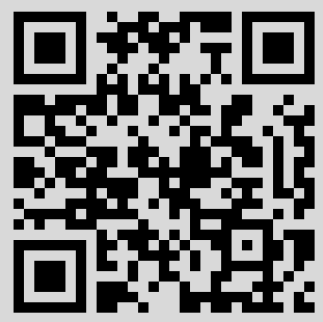




\title{
ИССЛЕДОВАНИЕ ЖИДКОКРИСТАЛЛИЧЕСКИХ СИСТЕМ С ПРИМЕСЯМИ МЕТОДАМИ МАТРИЧНОГО ФОРМАЛИЗМА
}

\begin{abstract}
С использованием матричного формализма исследуется жидкокристаллическая система, содержащая примеси. В качестве примера вычисляются статистические корреляторы флуктуаций ориентации директора и параметра порядка, характеризующего изотропную подсистему.
\end{abstract}

Ключевые слова: жидкий кристалл, примеси, статистический коррелятор.

Ранее предлагался формальный подход, позволяющий анализировать корреляционное поведение жидких систем, состоящих из большого числа компонент [1]. Такая методика с минимальными изменениями может быть расширена и на случай, когда жидкость характеризуется векторным параметром порядка. Наиболее показательным в этом смысле является жидкокристаллическая система. В нематической фазе жидкий кристалл, как известно, характеризуется единичным вектором-директором, определяющим направление преимущественной ориентации длинных осей молекул жидкого кристалла. Хотя параметром порядка для нематического жидкого кристалла является тензорная величина, но если исследовать малые отклонения директора от равновесного однородного распределения, то этот вектор малых флуктуаций директора можно выбрать в качестве параметра порядка, и система, таким образом, может быть описана векторным параметром порядка. Рассмотрим такую систему с примесями - жидкость в изотропной фазе. Будем предполагать, что жидкий кристалл и изотропные примеси образуют две взаимодействующие подсистемы, характеризующиеся директором и отклонением плотности от среднего значения, соответственно.

Как известно, для нематического жидкого кристалла энергия деформации, связанная с неоднородностью распределения директора, дается выражением [2]

$$
F_{n}=\frac{1}{2} \int\left(K_{11}(\operatorname{div} \vec{n})^{2}+K_{22}(\vec{n} \operatorname{rot} \vec{n})^{2}+K_{33}(\vec{n} \times \operatorname{rot} \vec{n})^{2}\right) d V,
$$

где $K_{i i}, i=1,2,3,-$ модули Франка, а $\vec{n}(\vec{r})$ - единичный вектор-директор.

\footnotetext{
*Киевский национальный университет, Киев, Украина. E-mail: vasilev@univ.kiev.ua
} 
Если система находится во внешнем поле (для определенности предположим, что это магнитное поле), следует учесть взаимодействие также и с ним. Энергия взаимодействия жидкокристаллической системы с магнитным полем $\vec{H}$ имеет вид

$$
F_{\mathrm{m}}=-\frac{1}{2} \chi_{a} \int(\vec{n} \vec{H})^{2} d V
$$

где через $\chi_{a}$ обозначена анизотропия магнитной восприимчивости.

Для изотропной подсистемы свободная энергия зависит от профиля скалярного параметра порядка $\phi(\vec{r})$, в качестве которого выберем локальную плотность изотропной фазы. Тогда свободная энергия изотропной подсистемы имеет вид [3]

$$
F_{\mathrm{i}}=\frac{1}{2} \int\left(a \phi^{2}+b(\operatorname{grad} \phi)^{2}\right) d V
$$

параметры $a$ и $b$ являются феноменологическими параметрами модели. Для простоты полагаем, что изотропная подсистема с магнитным полем не взаимодействует, однако необходимо учесть взаимодействие жидкокристаллической и изотропной подсистем. В общем случае энергия $F_{\text {in }}$ такого взаимодействия зависит как от профиля директора $\vec{n}(\vec{r})$, так и от скалярного параметра порядка $\phi(\vec{r})$, т.е. $F_{\text {i } n}=F_{\text {i } n}(\vec{n}(\vec{r}), \phi(\vec{r}))$.

Поскольку мы рассматриваем микроскопические примеси, есть все основания полагать, что их наличие на равновесное распределение $\vec{n}_{0}$ директора не влияет. Очевидно тогда, что директор будет направлен по магнитному полю, и это направление мы выберем в качестве $z$-оси координатной системы. Рассмотрим тепловые флуктуации директора. Для этого представим директор в виде $\vec{n}(\vec{r})=\vec{n}_{0}+\delta \vec{n}(\vec{r})$, где $\delta \vec{n}(\vec{r})=\left(\delta n_{x}, \delta n_{y}, 0\right)-$ вектор отклонения. Далее выполним разложение в ряд по параметрам $\delta n_{x}, \delta n_{y}$ и $\phi$ выражения для полной энергии

$$
F=F_{n}+F_{\mathrm{m}}+F_{\mathrm{i}}+F_{\mathrm{i} n} .
$$

Для объемной энергии деформации добавка, связанная с флуктуациями ориентации директора, задается формулой

$$
\begin{aligned}
\delta F_{n}=\frac{1}{2} & \int\left(K_{11}\left(\frac{\partial \delta n_{x}}{\partial x}+\frac{\partial \delta n_{y}}{\partial y}\right)^{2}+\right. \\
& \left.+K_{22}\left(\frac{\partial \delta n_{y}}{\partial x}-\frac{\partial \delta n_{x}}{\partial y}\right)^{2}+K_{33}\left[\left(\frac{\partial \delta n_{x}}{\partial z}\right)^{2}+\left(\frac{\partial \delta n_{y}}{\partial z}\right)^{2}\right]\right) d V .
\end{aligned}
$$

Слагаемое, связанное с энергией взаимодействия с магнитным полем, имеет вид

$$
\delta F_{\mathrm{m}}=\frac{1}{2} \chi_{a} H^{2} \int\left(\delta n_{x}^{2}+\delta n_{y}^{2}\right) d V
$$

а флуктуационная добавка к энергии изотропной примесной подсистемы есть

$$
\delta F_{\mathrm{i}}=\frac{1}{2} \int\left(a \delta \phi^{2}+b(\operatorname{grad}(\delta \phi))^{2}\right) d V,
$$


где $\delta \phi-$ флуктуация скалярного параметра порядка. Далее остается записать выражение для добавки к энергии взаимодействия подсистем, обусловленной флуктуациями ориентации директора и скалярного параметра порядка. Будем исходить из предположения, что градиент скалярного параметра порядка играет роль ориентирующего поля по отношению к директору. Это означает, что такая добавка к энергии зависит от производных флуктуаций скалярного параметра $\delta \phi$ и компонент отклонения $\delta n_{x}$ и $\delta n_{y}$ директора. Рассмотрим упомянутую добавку в следующем виде:

$$
\delta F_{\mathrm{i} n}=\int\left(W_{\|}\left(\delta n_{x}+\delta n_{y}\right) \frac{\partial \delta \phi}{\partial z}+W_{\perp}\left(\delta n_{x} \frac{\partial \delta \phi}{\partial x}+\delta n_{y} \frac{\partial \delta \phi}{\partial y}\right)\right) d V,
$$

где параметры $W_{\|}$и $W_{\perp}$ характеризуют взаимодействие подсистем.

Для дальнейшего анализа выполним преобразование Фурье. В результате получим для флуктуационных добавок к энергии следующие выражения:

$$
\begin{aligned}
\delta F_{n}= & \frac{1}{2 V} \sum_{\vec{q}}\left(K_{11}\left[q_{x}^{2}\left|\delta n_{x}(q)\right|^{2}+q_{y}^{2}\left|\delta n_{y}(q)\right|^{2}+q_{x} q_{y}\left(\delta n_{x}^{*}(q) \delta n_{y}(q)+\delta n_{x}(q) \delta n_{y}^{*}(q)\right)\right]+\right. \\
& \quad+K_{22}\left[q_{x}^{2}\left|\delta n_{y}(q)\right|^{2}+q_{y}^{2}\left|\delta n_{x}(q)\right|^{2}-q_{x} q_{y}\left(\delta n_{x}(q)^{*} \delta n_{y}(q)+\delta n_{x}(q) \delta n_{y}(q)\right)^{*}\right]+ \\
& \left.\quad+K_{33} q_{z}^{2}\left[\left|\delta n_{x}(q)\right|^{2}+\left|\delta n_{y}(q)\right|^{2}\right]\right), \\
\delta F_{\mathrm{m}}= & \frac{1}{2} \chi_{a} H^{2} \sum_{\vec{q}}\left(\left|\delta n_{x}(q)\right|^{2}+\left|\delta n_{y}(q)\right|^{2}\right), \\
\delta F_{\mathrm{i}}= & \frac{1}{2 V} \sum_{\vec{q}}\left(a+b q^{2}\right)|\delta \phi(q)|^{2}, \\
\delta F_{\mathrm{i} n}= & \frac{i}{2 V} \sum_{\vec{q}}\left(\left(W_{\perp} q_{x}+W_{\|} q_{z}\right)\left(\delta n_{x}^{*}(q) \delta \phi(q)-\delta n_{x}(q) \delta \phi^{*}(q)\right)+\right. \\
& \left.\quad+\left(W_{\perp} q_{y}+W_{\|} q_{z}\right)\left(\delta n_{y}^{*}(q) \delta \phi(q)-\delta n_{y}(q) \delta \phi^{*}(q)\right)\right)
\end{aligned}
$$

где $V$ - объем системы. Если ввести в рассмотрение вектор $\vec{\eta}(q)$ с координатами $\delta n_{x}(q), \delta n_{y}(q), \delta \phi(q)$, то выражение для полной энергии можно представить в виде (символ “+” использован для обозначения операции эрмитова сопряжения)

$$
\delta F=\frac{1}{2 V} \vec{\eta}^{+}(q) \hat{A}(q) \vec{\eta}(q),
$$

где элементы матрицы $\hat{A}(q)$ таковы:

$$
\begin{gathered}
A_{11}(q)=K_{11} q_{x}^{2}+K_{22} q_{y}^{2}+K_{33} q_{z}^{2}+\chi_{a} H^{2}, \\
A_{22}(q)=K_{11} q_{y}^{2}+K_{22} q_{x}^{2}+K_{33} q_{z}^{2}+\chi_{a} H^{2}, \\
A_{33}(q)=a+b q^{2}, \quad A_{12}(q)=A_{21}(q)=q_{x} q_{y}\left(K_{11}-K_{22}\right), \\
A_{13}(q)=A_{31}(q)=i\left(W_{\perp} q_{x}+W_{\|} q_{z}\right), \quad A_{23}(q)=A_{32}(q)=i\left(W_{\perp} q_{y}+W_{\|} q_{z}\right) .
\end{gathered}
$$

Для анализа статистических корреляций в системе выполним преобразование вида

$$
\vec{\eta}(q)=\widehat{S}(q) \vec{\xi}(q)
$$


В этом случае получим, что

$$
\delta F=\frac{1}{2 V} \vec{\xi}^{+}(q) \widehat{B}(q) \vec{\xi}(q)
$$

где

$$
\widehat{B}(q)=\widehat{S}^{+}(q) \hat{A}(q) \widehat{S}(q) .
$$

Матрицу перехода $\widehat{S}(q)$ выберем так, чтобы матрица $\widehat{B}(q)$ была диагональной. Если через $\vec{e}_{k}(q), \quad k=1,2,3$, обозначить собственные векторы матрицы $\hat{A}(q)$ единичной длины, то матрицу $\widehat{S}(q)$ можно представить как прямую сумму этих векторов:

$$
\widehat{S}(q)=\vec{e}_{1}(q) \oplus \vec{e}_{2}(q) \oplus \vec{e}_{3}(q)
$$

Если собственные числа $\lambda_{k}(q)$ соответствуют собственным векторам $\vec{e}_{k}(q)$, то именно эти собственные числа будут размещены на диагонали матрицы $\widehat{B}(q)$. Это означает, что полная энергия будет иметь вид

$$
\delta F=\frac{1}{2 V} \sum_{k=1}^{3} \lambda_{k}(q)\left|\vec{\xi}_{k}(q)\right|^{2}
$$

где $\vec{\xi}_{k}(q)$ есть $k$-я компонента вектора $\vec{\xi}(q)$. Из этого выражения, используя теорему о равнораспределении энергии по степеням свободы, легко получить

$$
\left\langle\left|\vec{\xi}_{k}(q)\right|^{2}\right\rangle=\frac{k_{B} T V}{\lambda_{k}(q)}
$$

Интерес представляют корреляторы $\left\langle\vec{\eta}_{i}\left(\vec{r}_{1}\right) \vec{\eta}_{j}\left(\vec{r}_{2}\right)\right\rangle$, которые в $\vec{q}$-пространстве соответствуют статистическим средним от произведения компонент вектора $\vec{\eta}(q)$. В частности, справедливо соотношение

$$
\left\langle\eta_{i}(q) \eta_{j}^{*}(q)\right\rangle=\sum_{k=1}^{3} S_{i k}(q) S_{k j}^{*}(q)\left\langle\left|\vec{\xi}_{k}(q)\right|^{2}\right\rangle=\sum_{k=1}^{3} e_{k}^{(i)}(q) e_{k}^{(j) *}(q)\left\langle\left|\vec{\xi}_{k}(q)\right|^{2}\right\rangle
$$

где через $e_{k}^{(i)}(q)$ обозначена $i$-я компонента собственного вектора $\vec{e}_{k}(q)$. Таким образом, задача сводится к расчету собственных векторов и собственных чисел матрицы $\hat{A}(q)$. Дальнейший анализ проведем для случая одноконстантного приближения $K_{11}=K_{22}=K_{33} \equiv K$. Положим также для простоты, что $W_{\perp}=0$. Это соответствует ситуации, когда жидкокристаллическая система взаимодействует только с флуктуациями скалярного параметра порядка в направлении внешнего магнитного поля.

При таких предположениях выражение для матрицы $\hat{A}(q)$ несколько упрощается:

$$
\hat{A}(q)=\left(\begin{array}{ccc}
K q^{2}+\chi_{a} H^{2} & 0 & i W_{\|} q_{z} \\
0 & K q^{2}+\chi_{a} H^{2} & i W_{\|} q_{z} \\
i W_{\|} q_{z} & i W_{\|} q_{z} & a+b q^{2}
\end{array}\right) .
$$


Несложные вычисления в этом случае дают собственные числа

$$
\begin{gathered}
\lambda_{1,2}(q)=\frac{1}{2}\left(a+(b+K) q^{2}+\chi_{a} H^{2} \pm \sqrt{\left(a+(b-K) q^{2}-\chi_{a} H^{2}\right)^{2}-8 W_{\|}^{2} q_{z}^{2}}\right), \\
\lambda_{3}(q)=K q^{2}+\chi_{a} H^{2} .
\end{gathered}
$$

Собственные векторы, соответствующие указанным выше собственным числам, равны

$$
\begin{gathered}
\vec{e}_{1,2}(q)=\frac{1}{\sqrt{\left(K q^{2}+\chi_{a} H^{2}-\lambda_{1,2}(q)\right)^{2}+2 W_{\|}^{2} q_{z}^{2}}}\left(\begin{array}{c}
i W_{\|} q_{z} \\
i W_{\|} q_{z} \\
\lambda_{1,2}(q)
\end{array}\right), \\
\vec{e}_{3}(q)=\left(\begin{array}{c}
1 / \sqrt{2} \\
-1 / \sqrt{2} \\
0
\end{array}\right) .
\end{gathered}
$$

Полученных выражений для собственных чисел и собственных векторов достаточно для того, чтобы вычислить любые представляющие интерес корреляторы. Хочется отметить, что среди найденных собственных чисел имеется $\lambda_{3}(q)$, которому отвечает постоянный, не зависящий от $q$ собственный вектор, что соответствует парным корреляциям в чистом нематическом жидком кристалле. Таких корреляторов для изотропной подсистемы в чистом виде нет. Однако это является следствием выбора типа и характера энергии взаимодействия подсистем.

Схожий метод использовался ранее при изучении чистых (т.е. без примесей) жидкокристаллических систем [4], [5]. Такой подход в той или иной реализации достаточно продуктивен, поскольку позволяет рассматривать разные жидкокристаллические системы (нематики, смектики или холестерики) в рамках общей концепции [5]. В данной работе этот универсальный формализм расширен на случай системы с примесями. Следует, правда, отметить, что в последнем случае реализация метода существенно зависит от выбранного типа взаимодействия подсистем. Тот, что был предложен выше, неплохо согласуется с существующими теоретическими и экспериментальными данными (см., например, [6]).

\section{Список литературы}

[1] А.Н. Васильев, ТMФ, 139 (2004), 331.

[2] П. де Жен, Физика жидких кристаллов, Мир, М., 1977.

[3] М.А. Анисимов, Критические явления в жидкостях и жидких кристаллах, Наука, М., 1987; А.З. Паташинский, В.Л. Покровский, Флуктуационная теория фазовых переходов, Наука, М., 1982.

[4] А. Ю. Вальков, В. П. Романов, А. Н. Шалагинов, УФН, 164:2 (1994), 149.

[5] А. Ю. Вальков, В. П. Романов, М. В. Романов, ЖЖЭТФ, 120:2(8) (2001), 389.

[6] P. K. Mukherjee, Liquid Crystals, 22:3 (1997), 239; А.Н. Васильев, И. П. Пинкевич, Т. Слуцкин, Писъма в ЖЖЭФ, 80:5 (2004), 372.

Поступила в редакцию 27.VII.2005 г., после доработки 17.X.2005 г. 